\title{
Detection of High-Risk Human Papillomavirus by Chromogenic in Situ Hybridization Method in Liquid-Based Cervicovaginal Cytology Specimens with Atypical Squamous Cells of Undetermined Significance
}

\author{
Melin Gecer \\ Bezmialem Foundation University Hospital Department of Pathology \\ Adnan menderes bulvarı, Vatan street Fatih-Istanbul,Turkey
}

\begin{abstract}
Background: Cervical cancer is the second most common cancer after breast cancer in women worldwide. Owing to comprehensive cervical screening programs, a significant decrease has been observed in the incidence of invasive cervical cancers. Human Papillomavirus (HPV) is the underlying etiology in $99.7 \%$ of the cases of cervical cancer and is a major risk factor for the development of precancerous and cancerous cervical lesions. Chromogenic in situ hybridization method (CISH) is one of the methods used to detect high risk HPV in liquid based smears. Aims: In this study, we conducted an archive search in the department of pathology and we used chromogenic in situ hybridization technique to investigate whether HPV DNA was present in liquid -based smears of 68 patients who had been found to have atypical squamous cells of undetermined significance (ASCUS) and whether a cervical intraepithelial lesion was present in the biopsy samples of patients who were positive for HPV DNA.Methods and Material:. We prepared additional samples from liquid -based preparations obtained by the ThinPrep method. The CISH procedure was performed on the Benchmark Automated Slide Stainer (Ventana) according to the manufacturer's standard protocol.Results and Conclusions: We found HPV DNA in the samples of 10 patients by using INFORM HPV III high risk $(16,18,31,33,35,39$, 45, 51, 52, 56, 58, 68, and 70) (Ventana, Tucson, AZ) probe, with CISH method on each additional sample, and biopsy results of the respective patients, indicated low grade squamous intraepithelial lesion (LGSIL) in 8 patients and high grade squamous intraepithelial lesion (HGSIL) in 2 cases.
\end{abstract}

Keywords: Human papillomavirus, chromogenic in situ hybridization, atypical squamous cells of undetermined significance

DOI: $10.7176 / \mathrm{JHMN} / 80-10$

Publication date:September $30^{\text {th }} 2020$

\section{INTRODUCTION}

Cervical cancer is the second most common cancer type after breast cancer, in women worldwide. In Turkey, cervical cancer is the third most common cancer after uterine and ovarian cancers according to the data of the Ministry of Health. ${ }^{[1]} \mathrm{A}$ significant decrease has been observed in the incidence of invasive cervical cancers, owing to comprehensive cervical cancer screening programs, ${ }^{[2]}$ Human Papillomavirus (HPV is the underlying agent in $99.7 \%$ of the cases of cervical cancer and is a major risk factor for the development of precancerous and cancerous cervical lesions. ${ }^{[1,2]}$ Smear screening tests are very important in early detection of cervical cancer and monitoring the progression of the disease. Although the PAP smear test that has been widely used worldwide to detect precancerous and cancerous lesions is paramount, the sensitivity of this test is limited. In addition to the PAP smear test, a variety of more sensitive molecular methods can be used to detect HPV DNA in tissues and cytological preparations. The signal amplification test (Hybrid capture), nucleic acid amplification test (PCR) and direct hybridization (In situ hybridization) are among these molecular methods ${ }^{[3,4]}$ In this study, HPV DNA was tested by chromogenic in situ hybridization method in liquid -based smear specimens of the patients who had been found to have atypical squamous cells of undetermined significance and we have investigated whether a cervical intraepithelial lesion is present in the biopsy specimens of patients who were positive for HPV DNA.

\section{MATERIALS AND METHODS}

We investigated HPV DNA in a total of 68 patients identified as having cervicovaginal specimens diagnosed with ASCUS during our search for cervicovaginal specimens in the archives of our department of pathology. We prepared additional samples from liquid -based preparations obtained by the ThinPrep method and we investigated HPV DNA in each sample by using INFORM HPV III high risk $(16,18,31,33,35,39,45,51,52$, 56, 58, 68, and 70) (Ventana, Tucson, AZ) probe with CISH method. The CISH procedure was performed on a Benchmark automated slide stainer (Ventana), according to the manufacturer's standard protocol. After the initial incubation periods and buffer rinses, an endopeptidase was applied to and incubated on the slides, permitting the exposure of nuclear DNA to the liquid-based prep high -risk reagent probe. The probe consisted of a cocktail of fluorescein-labeled DNA oligonucleotides that had been demonstrated to hybridize to specific 
high -risk HPV genotypes. Initially, a Mouse anti-fluorescein primary antibody bound the probe. Then, a biotinylated secondary horse antibody formulation bound to the primary Mouse antibodies. This step was followed by the addition of a streptavidin enzyme (alkaline phosphatase) conjugate that bound to the biotin present on the secondary antibody. The specific secondary antibody streptavidin enzyme complex then was visualized by light microscopy using nitroblue tetrazolium as a substrate for a precipitating enzyme-generated product. As the result of successful probe hybridization, deep blue to black nuclear staining was present in the affected cells. A nuclear fast red-based counterstain was used. Positive control slides consisted of commercially available prepared cytocentrifuged slides using a CaSki cell line preparation containing approximately 400 copies of HPV type 16 DNA per cell (Ventana). Negative control slides were prepared in house from the cellular residua of liquid based cervicovaginal samples that had been diagnosed cytomorphologycally as no intraephitelial lesion or malignancy. The CISH slides were screened by 2 cytotechnologists who referred all potentially positive slides for definitive interpretation. A positive result was defined as any convincing blue to black nuclear staining, whether focal or global within the given nucleus. Convincing staining within a single cell was interpreted as positive per manufacturer guidelines. In situ hybridization signal was evaluated in three types. Type I) diffuse (episomal), Type II) Punctate (integrated), Type III) Combined (episomal and integrated). The episomal staining was associated with low grade squamous intraepithelial lesion (LGSIL) and punctate staining was associated with invasive carcinoma. ${ }^{[5,6]}$ All testing was performed in a blinded manner. All patients who had HPV DNA underwent a colposcopy exam and cervical biopsy and the biopsy specimens were evaluated for the presence of any squamous intraepithelial lesion. In addition, 25 patients who were negative for HPV DNA, also underwent colposcopy and 12 patients who were suspected for the presence of a squamous intraepithelial lesion had a cervical biopsy.

\section{RESULTS}

The ages of 68 patients who had been diagnosed with ASCUS (figure 1) varied between 24 and 66 (average age 40.9 years) years. While $81.1 \%$ of these patients were premenopausal women, $19.9 \%$ of them were postmenopausal. HPV DNA was detected in 10 out of 68 patients via the CISH method (14.7\%). 14.3\% of premenopausal patients were positive for HPV while this rate was $15.4 \%$ among postmenopausal women. There was no significant difference between premenopausal and postmenopausal patients in terms of having a positive HPV test result (p: 0.10) (Chi-Square Tests). A squamous intraepithelial lesion was seen in all biopsy samples taken from the patients who had HPV DNA based on the tests conducted in liquid -based smears. While low grade squamous intraepithelial lesions were found in 8 cases $(80 \%)$, high grade squamous intraepithelial lesions were found in 2 cases (\%20). A type 1 signal (diffuse, episomal) (figure 2) staining was found in 6 patients while there was a type 3 signal (combined: episomal and integrated) (figure 3-4) staining in 4 patients. Episomal signal staining was present in 6 LSIL cases (6/8), while combined staining was assessed in the remaining two LSIL cases $(2 / 8)$. Combined staining was evident in both of the HSIL cases. No squamous intraepithelial lesion was found in the biopsy samples of 12 patients who had no HPV DNA detected in their cytology preparations.

\section{DISCUSSION}

HPV infection is a significant risk factor for pre-invasive and invasive cervical cancers. HPV infection, which is sexually-transmitted and common in the society, is seen in almost $99.7 \%$ of the cases of cervical cancer, and it is held responsible for anal, penile and vulvar cancers. ${ }^{[5]} \mathrm{HPV}$ is a double helical DNA virus and over 200 subtypes have been found. About 40 of them cause infection in the human genital system. In terms of their oncogenic potentials, genital HPV types are classified in two groups which are high risk and low risk. While high risk HPV types $(16,18,31,33,35,39,45,51,52,56,58,59,68,73$, and 82) have a role in carcinogenesis, low risk HPV types $\left(6,11,42,43\right.$, and 44) cause benign lesions such as condyloma acuminatum. ${ }^{[6,7]}$ The prevalence of HPV peaks between 25 and 35 years of age, which is the period when sexual contact is the highest. Cytological abnormalities are found only in about $5 \%$ of the individuals infected with HPV, with a high rate at younger ages. ${ }^{[8]}$ The review of the Turkish medical literature for ASCUS Pap smears tested for HPV yielded different results. In a study conducted by Dursun et al, the rate of ASCUS smears positive for HPV was reported as $37 \%$ while in another study it was reported to be $22 \%{ }^{\left[{ }^{[8,9]}\right.}$ In our study, the rate of HPV DNA positive women did not differ between the pre- and postmenopausal groups, however the sample size was small. In this study, we preferred the CISH method since it can be performed under light microscopy and it is easy to evaluate. ${ }^{[10]} \mathrm{We}$ found that $14.7 \%$ of the patients tested positive for HPV DNA. While a LGSIL was present in $80 \%$ of these patients, a HGSIL was identified in the remaining 20\%. Patients who tested negative for HPV DNA underwent a colposcopy examination, and a biopsy was conducted in 12 patients who were suspicious for a malignant process. However, no lesion was found in the biopsies of these patients. No pathological findings were detected during the 2-year smear follow-ups in patients who did not undergo a biopsy.

The follow-up strategies for patients who are found to have ASCUS according to Bethesta system include direct colposcopy examination, follow-up smears at 6-month intervals or HPV checks in patients who have high 
oncogenic risk. Previous studies, revealed that the addition of molecular tests detecting high-oncogenic risk HPV to cytology tests increased the sensitivity to detect cervical intraepithelial neoplasia and decreased the rate of unnecessary colposcopy exams. ${ }^{[1,12]}$ The HPV test can also show us the progression of the disease; so it has a prognostic significance. For example, the possibility of developing cervical dysplasia or cancer within 5-10 years in a woman with a normal cytology, who is negative for HPV is very low. Therefore, screening range can be extended to those who have negative results for the HPV test. On the other hand, in case of the presence of a high risk HPV in women who had a cervical intraepithelial lesion in the pap smear test (and if it is also confirmed with biopsy), the risk for developing a CIN 3 or more advanced lesion (cancer) within following two years is between 60 to $80 \%$. Therefore, even the HPV test is not used in screening, it is utilized for directing (in triage of) patients with cervical dysplasia. Using the HPV test in this context also decreases the numbers of unnecessary colposcopy exams and biopsies. ${ }^{[13]}$

Our series revealed that low or high grade intraepithelial lesions were present only in HPV DNA positive patients and none of the HPV DNA negative patients had an intraepithelial lesion and this result further support this conclusion.

\section{References}

1. Akyar I, Aydın Ö, Yakıcıer MC, Kocagöz ZT, Ince Ü at all.2013.Human papillomavirus prevalence and type in liquid-based cervical samples from.Turkish women in a selected risk group. Turk J Med Sci.43:96370

2. Schiller CL, Nickolov AG, Kaul KL, Hahn EA, Hy JM, et al. 2004. High-risk human papillomavirus detection: a split-sample comparison of hybrid capture and chromogenic in situ hybridization. Am J Clin Pathol 121:537-45.

3. Samama B, Plas-Roser S, Schaeffer C, Chateau D, Fabre M.2002. HPV DNA Detection by In Situ Hybridization with Catalyzed Signal Amplification on Thin-layer Cervical Smears. J Histochem Cytochem 5010:1417-20.

4. Guo M, Gong Y, Deavers M, Silva EG, Jan YJ, et al. 2008.Evaluation of a commercialized in situ hybridization assay for detecting human papillomavirus DNA in tissue specimens from patients with cervical intraepithelial neoplasia and cervical carcinoma. J Clin Microbiol 46:274-80.

5. Lee JU, Shin JH, Kim JO, Choi YJ, Lee KY, et al.2010. Evaluation of the HPV ISH Assay in Cervical Cancer. Korean J Pathol 44:513-20.

6. Yousefzadeh A, Mostafavizadeh SM, Jarollahi A, Raeisi M, Garshasbi M, et al.2014. Human papillomavirus (HPV) prevalence and types among women attending regular gynecological visit in Tehran, Iran. Clin Lab 60:267-73.

7. Augusto EF, Santos LS, Oliveira LHS. 2014.Human papillomavirus detection in cervical scrapes from women attended in the Family Health Program. Rev. Latino-Am. Enfermagem 22:100-7.

8. Dursun P1, Senger SS, Arslan H, Kuşçu E, Ayhan A.2009. Human papillomavirus (HPV) prevalence and types among Turkish women at a gynecology outpatient unit. BMC Infect Dis. 30;9:191.

9. Dursun P, Ayhan A, Mutlu L, Çağlar M, Haberal A, et al.2013 HPV types in Turkey: multicenter hospital based evaluation of 6388 patients in Turkish gynecologic oncology group centers. Turk Patoloji Derg 29:210-6

10. Bartlett JM, Campbell FM, Ibrahim M, Wencyk P, Ellis I, , et al.2009. Chromogenic in situ hybridization: a multicenter study comparing silver in situ hybridization with FISH. Am J Clin Pathol 132:514-20.

11. Schiffman M, Adrianza ME.2000. ASCUS-LSIL triage study, design, methods and characteristics of trial participants. Acta Cytol 44:726-42.

12. Schiffman M, Solomon D. 2003.Findings to date from the ASCUS-LSIL triage study (ALTS). Arch Pathol Lab Med 127:946-9.

13. Lester JL, Qureshi MN.2005. HPV DNA Testing in the Triage of Atypical Squamous Cells of Undetermined Significance (ASCUS): Cost Comparison of Two Methods. Diagn Cytopathol 33:2. 
Figure1. ASCUS in liquid-based cervicovaginal samples (HEX400)

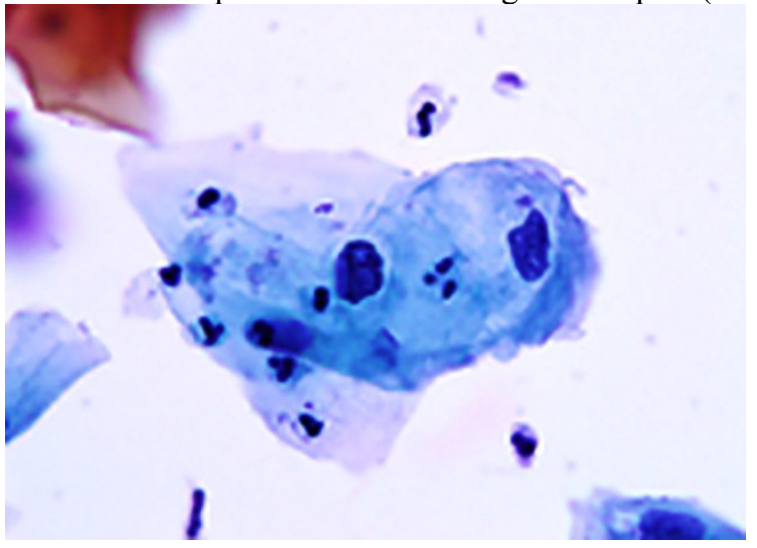

Figure2. Positive chromogenic in situ hybridization:diffüse (episomal ) patern (deep blue nuclear stain of infected cells) (İnform HPVIII;magnificationX400)

Figure3, ASCUS liquid-based smear showing both diffuse and punctate staining in squamous metaplasia cells (İnform HPVIII;magnificationX400)

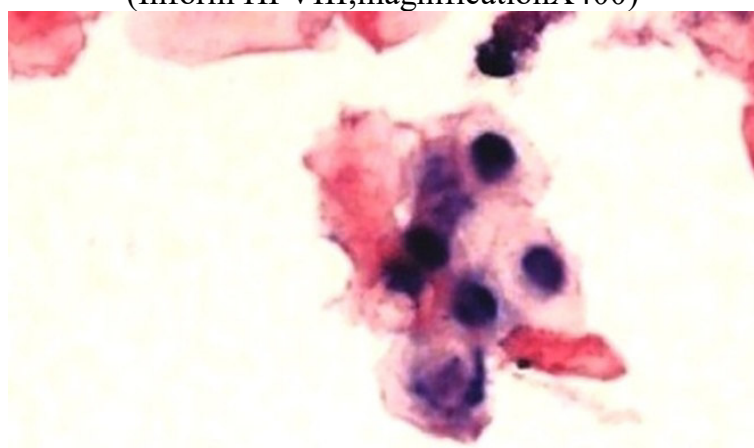


Figure4. ASCUS liquid-based smear showing punctate staining. ((İnform HPVIII;magnificationX400)

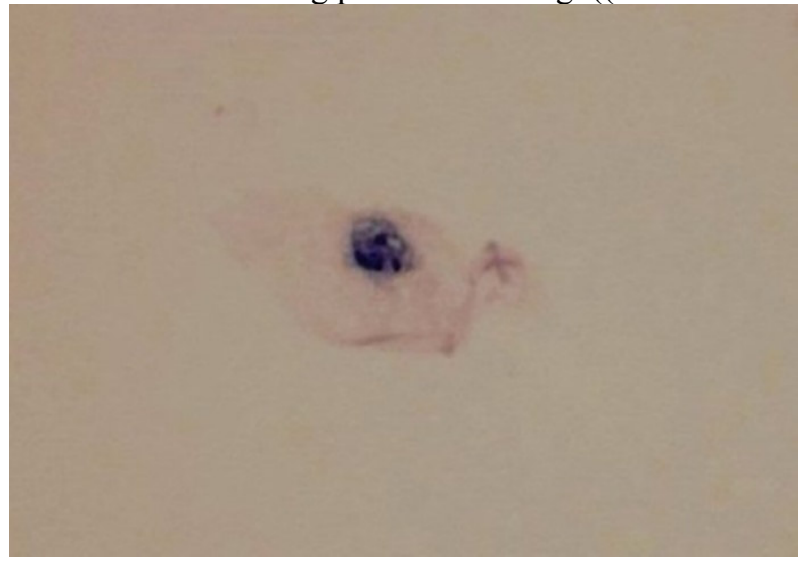

Figure5-Control slides stained with INFORM HPV III Family 16 Probe (C).

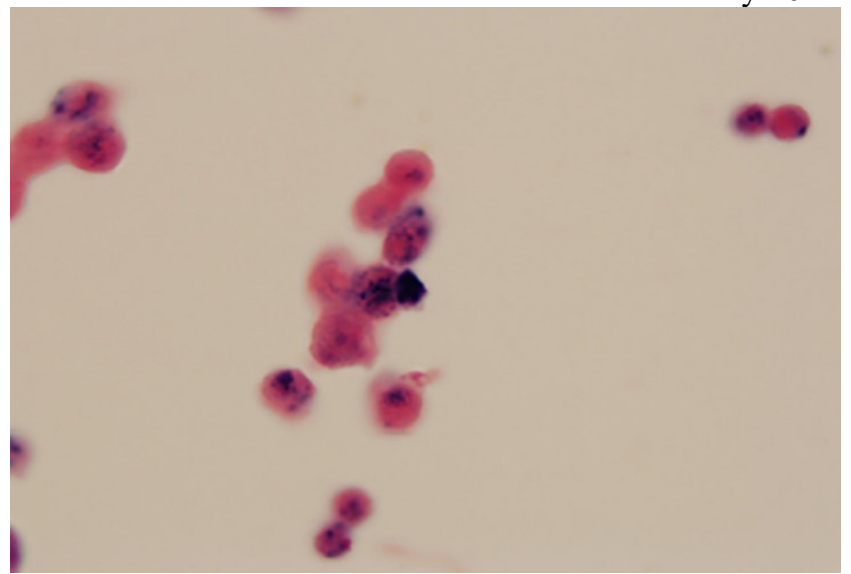

